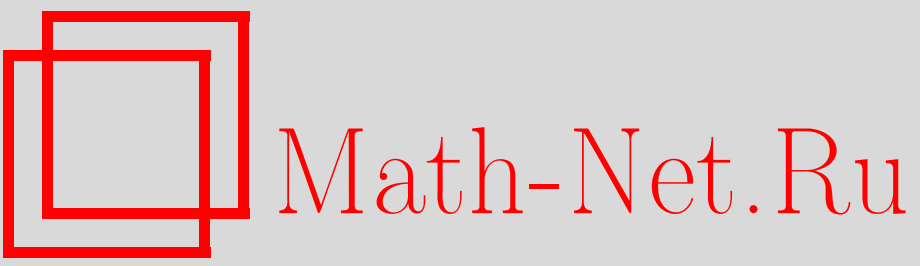

А. Д. Миронов, Интегрируемость в теориях струн/поля и гамильтоновы потоки в пространстве физических систем, ТМФ, 2003, том 135, номер 3, 434-451

DOI: https://doi.org/10.4213/tmf200

Использование Общероссийского математического портала Math-Net.Ru подразумевает, что вы прочитали и согласны с пользовательским соглашением

http://www.mathnet.ru/rus/agreement

Параметры загрузки:

IP : 3.91 .87 .62

26 апреля 2023 г., 13:24:26 


\section{ИНТЕГРИРУЕМОСТЬ В ТЕОРИЯХ СТРУН/ПОЛЯ И ГАМИЛЬТОНОВЫ ПОТОКИ В ПРОСТРАНСТВЕ ФИЗИЧЕСКИХ СИСТЕМ}

Как известно, интегрируемость в теориях струн/поля возникает при рассмотрении динамики на пространстве модулей физических теорий. Это подразумевает, что нужно изучать динамику по необычному времени, подобному константе связи или другим величинам, параметризующим конфигурационное пространство физических теорий. Динамика, задаваемая изменением констант связи, может рассматриваться как каноническое преобразование или, инфинитезимально, как гамильтонов поток в пространстве физических систем. Здесь кратко рассмотрен пример интегрируемых механических систем. Тогда любая функция $T(\vec{p}, \vec{q})$ задает однопараметрическое семейство интегрируемых систем в окрестности одной системы. В случае интегрируемой системы с несколькими константами связи соответствующие "гамильтонианы" $T_{i}(\vec{p}, \vec{q})$ удовлетворяют уравнениям Уизема, а после квантования первоначальной системы становятся операторами, удовлетворяющими условию нулевой кривизны в пространстве констант связи.

Ключевые слова: теория струн, квантовая теория поля, интегрируемые системы.

\section{1. ВВЕДЕНИЕ}

Один из основных уроков теории струн состоит в том, что, в противоположность подходу обычной теории поля, следует изучать теорию не при фиксированных значениях параметров, а наоборот, варьировать как можно больше параметров, для того чтобы обнаруживать новые скрытые структуры теории. Большинство таких структур, обнаруженных за последние годы (интегрируемость, дуальность, топологические свойства и т.д. теорий поля/струн), просто и не может быть открыто при фиксированных значениях параметров. Более того, если теория ограничена некоторыми дополнительными требованиями (что часто случается в обычной теории поля, где, как правило, требуют перенормируемости, унитарности и т.д.), простых структур в ней обычно либо не бывает, либо они реализуются очень сложным, нелинейным образом. Эта ситуация очень напоминает интегрируемые системы, которые часто описываются как простые свободные

${ }^{*}$ Физический институт им. П.Н. Лебедева РАН, Институт теоретической и экспериментальной физики, Москва, Россия. E-mail: mironov@lpi.ac.ru, mironov@itep.ru 
системы на некотором многообразии, но приобретают нетривиальную динамику после гамильтоновой редукции на фазовом пространстве.

Среди новых интересных структур, обнаруженных описанным путем, - интегрируемость полных эффективных действий в квантовой теории поля (см., например, [1]). Под полным эффективным действием мы подразумеваем производяшую функцию всех корреляционных функций. Оказывается, что это же самое эффективное действие обладает также рядом других интересных свойств, обычно топологической или подобной природы.

В настояшей работе в разделах 2 и 3 мы перечисляем несколько типичных примеров структур такого типа. Однако следует заметить, что список примеров далеко не исчерпьвается эффективными действиями. Иногда приходится иметь дело с более сложными величинами, подобными метрике $\sigma$-модели, описывающей низкоэнергетическое эффективное действие, как, например, в случае теории Виттена-Зайберга, обсуждаемом в разделах 4 и 5. В разделах 6 и 7 мы развиваем, следуя [2], формализм, который мог бы объяснить все наблюдаемые структуры эффективных теорий, в частности мы предлагаем некоторое объяснение интегрируемости эффективных действий. Наконец, в разделах 8 и 9 приведены явные примеры.

\section{2. МАТРИЧНАЯ МОДЕЛЬ КАК ПРОСТЕЙШИЙ ПРИМЕР}

Рассмотрим простейший пример матричного интеграла, который имитирует интеграл по траекториям теории поля. Этот пример возникает в теории некритических струн [3]. Именно, мы рассматриваем интеграл по эрмитовой матрице размера $N \times N$ при соответствуюшей мере Хаара. Статистическая сумма этой теории в простейшем случае дается интегралом

$$
Z_{N}(g)=\int d M e^{\operatorname{Tr} M^{2}+g \operatorname{Tr} M^{3}}
$$

Первый член в экспоненте является аналогом кинетического члена, в то время как второй генерирует кубическое взаимодействие. Следовательно, можно изучать этот интеграл с помощью теории возмушений по параметру $g$. Здесь мы ограничиваемся кубическим взаимодействием, для того чтобы воспроизвести ситуацию в теории поля, где обычно допустимо конечное число различных (полиномиальных) взаимодействий.

Если теперь захотеть узнать что-либо об этом интеграле, нужно деформировать теорию так, чтобы включить в нее больше взаимодействий, а также допустить вариацию констант в интеграле (1). Пусть размер $N$ матрицы будет переменным, в то время как другая переменная $t$ будет коэффициентом перед линейным членом в потенциале, который мы полагали в (1) равным нулю. Тогда можно легко показать [4], что статистическая сумма как функция этих двух параметров удовлетворяет разностно-дифференциальному уравнению

$$
\frac{\partial^{2} \phi_{N}}{\partial t^{2}}=e^{\phi_{N+1}-\phi_{N}}-e^{\phi_{N}-\phi_{N-1}}, \quad e^{\phi_{N}} \equiv \frac{Z_{N+1}}{Z_{N}} .
$$


В этом уравнении можно узнать уравнение цепочки Тоды, причем $Z_{N}$ является ее $\tau$-функцией [5]. Цепочка Тоды является интегрируемой системой, следовательно, в системе существует (бесконечно) много сохраняющихся величин в инволюции, каждая из которых может быть взята в качестве гамильтониана, т.е. каждая из них генерирует некоторое время. Поскольку все эти гамильтонианы находятся в инволюции, имеется (бесконечно) много коммутируюших временных потоков, которые приводят к целой иерархии уравнений движения. Эта иерархия называется иерархией цепочки Тоды. Заметим, что все уравнения иерархии описьваются одной функцией бесконечного числа переменных времени, которая называется $\tau$-функцией. Мы ожидаем, что эта $\tau$-функция должна быть статистической суммой нашей системы.

Что представляют собой эти другие переменные времени? В действительности они имеют очень простой смысл: это не что иное, как другие константы связи, при членах $\operatorname{Tr} M^{k}$ [4]. Таким образом, для того чтобы получить полную интегрируемую систему, нужно рассмотреть все односледовые взаимодействия в (1), причем соответствующие константы связи являются переменными времени в иерархии цепочки Тоды, а статистическая сумма является $\tau$-функцией цепочки Тоды. Заметим, что эта статистическая сумма является производящей функцией всех корреляторов в теории.

В действительности введение бесконечного числа взаимодействий позволяет найти некоторые другие структуры помимо интегрируемости. Скажем, если сделать полиномиальную замену переменных в (1) с потенциалом, содержащим все односледовые операторы, это приведет [6] к следующему набору условий (тождеств Уорда), которым удовлетворяет статистическая сумма:

$L_{n}\left(\int d M \exp \left(\sum_{k} t_{k} \operatorname{Tr} M^{k}\right)\right)=0, \quad n \geqslant-1, \quad L_{n} \equiv \sum_{k} k t_{k} \frac{\partial}{\partial t_{k+n}}+\sum_{k=0}^{n} \frac{\partial^{2}}{\partial t_{n-k} \partial t_{k}}$.

Эти условия образуют борелевскую подалгебру алгебры Вирасоро. Заметим, что они существенно включают полный набор времен и, следовательно, не могут быть найдены при “неполном" действии, подобном (1) (в противоположность интегрируемости, которую мы частично наблюдали уже при двух параметрах). Действительно, тождества Уорда содержат в себе более тонкую информацию о теории, в конечном итоге связанную с точной ренормгруппой (ренормгруппой Полчинского) [7]. По этой причине их решения обычно однозначно задают статистическую сумму [8].

\section{3. ИНТЕГРИРУЕМОСТЬ В ТЕОРИЯХ СТРУН/ПОЛЯ}

Итак, основной урок, который можно извлечь из приведенного простого примера, состоит в том, что следует добавлять как можно больше ненулевых констант связи и изучать вариации по ним. Тогда, к примеру, в случае полного эффективного действия (т.е. для производящей функции всех корреляторов) можно найти ряд фундаментальных уравнений, подобных интегрируемости, полному набору тождеств Уорда и т.д.

Заметим, что само явление классической интегрируемости в различных квантовых системах наблюдалось многократно. Примеры в основном имеются среди квантовых 
интегрируемых систем просто потому, что только эти системы могли быть точно решены и интегрируемость в них могла быть апостериори обнаружена. Однако найденные примеры взяты из различных областей (двумерные квантовые теории поля, решеточные статистические суммы и т.д. [9]-[11]), что наводит на мысль, что это явление очень обшее. Более того, оно также наблюдалось в матричных моделях совершенно другого типа (так называемые непрерывные матричные модели [12]), в двумерных калибровочных теориях [13] и т.д. Все эти примеры двумерные, однако ниже мы также упомянем примеры в старших размерностях. Более того, в рамках теории струн также уже давно ожидается, что сумма всего пертурбативного ряда для амплитуд (и статистической суммы) может быть описана некоторой квантовой интегрируемой системой [14], [1]. Таким образом, сам факт, что эффективное действие для көантовой системы является $\tau$ - функцией некоторой классической интегрируемой системы ${ }^{1)}$, известен уже много лет, хотя до сих пор не был объяснен. В разделе 6 мы, следуя [2], предлагаем его некоторое объяснение.

Однако эффективные действия зависят не только от констант связи. Другими параметрами, существенно определяющими теорию, являются граничные условия [16] или, что по сути то же самое, вакуумные средние различных полей. Оказывается, учет этих параметров делает общие структуры теории значительно богаче. Удивительно, что интегрируемость присутствует и в этом случае, хотя и реализуется другим образом.

\section{4. ИНТЕГРИРУЕМОСТЬ В ТЕОРИИ ВИТТЕНА-ЗАЙБЕРГА}

Базовым примером эффективного действия, зависящего от вакуумных средних, является низкоэнергетическое эффективное действие $N=2$ суперсимметричных калибровочных теорий в размерностях 4,5 и 6 , содержаших различные поля материи. Точное решение для такого действия было найдено Зайбергом и Виттеном [17], в то время как его интегрируемые свойства были обнаружены в [18] (многочисленные обсуждения решений Виттена-Зайберга и их интегрируемых структур можно найти в книге [19]). Говоря более конкретно, изучается теория с калибровочной группой $G$ с симметрией, спонтанно нарушенной до $U(1)^{n}$, с $n$ скалярными полями, имеющими ненулевые вакуумные средние $\left\langle\phi_{i}\right\rangle=a_{i}$, и с $n$ безмассовыми фотонами (кулоновская ветвь). Тем самым при низких энергиях эффективно получается $n N=2$ суперсимметричных

\footnotetext{
1) Интересно, что все примеры могут быть разделены на два больших класса систем, естественным образом зависящих либо от времен (констант связи), либо от так называемых переменных Мивы, которые просто являются собственными значениями некоторой (бесконечной) внешней матрицы. Примером системы первого типа, к которому принадлежат и матричные модели (1), является квантовая модель нелинейного уравнения Шредингера, производящий функционал корреляторов которой является $\tau$-функцией некоторого классического нелинейного уравнения того же типа (нелинейного уравнения Шредингера) [10]. Типичный пример другого типа дается статистической суммой шестивершинной модели с нетривиальными граничными условиями. Эта статистическая сумма оказывается $\tau$-функцией двумеризованной системы Тоды, переписанной в переменных Мивы [11]. Другим типичным представителем того же класса теорий являются непрерывные матричные модели [12] [13]. В частности, такие матричные модели имеют прямое отношение к пределу очень низких энергий в КХД [15].
} 
фотодинамик. Скалярная часть действия может быть описана $\sigma$-моделью с метрикой, которая является второй производной некоторой (единственной) голоморфной функции $T_{i j}(\phi)=\partial_{i, j}^{2} \mathcal{F}(\phi)$, называемой препотенциалом. Сингулярности этой функции известны из физических аргументов (дуальности и согласованности с ренормгрупповыми потоками) [17] и фиксируют $\mathcal{F}(\phi)$. Препотенциал полностью фиксирует точные низкоэнергетические амплитуды в теории. Он может быть вычислен следуюшим образом.

Прежде всего нужно найти подходящие переменные, модулярные свойства которых согласуются с полевой интерпретацией. Эти переменные - интегралы мероморфной 1-формы $d S$ вдоль циклов на некоторой двумерной римановой поверхности $a_{i}$ и $a_{i}^{\mathrm{D}}:$

$$
a_{i}=\oint_{A_{i}} d S, \quad a_{i}^{\mathrm{D}}=\oint_{B_{i}} d S,
$$

где $i=1, \ldots, N_{c}-1$ для калибровочной групшы $S U\left(N_{c}\right)$.

Эти интегралы играют двоякую роль в подходе Виттена-Зайберга. Во-первых, можно вычислять препотенщиал $\mathcal{F}$ и, следовательно, низкоэнергетическое действие, идентифицируя $a^{\mathrm{D}}$ с $\partial \mathcal{F} / \partial a$ при том, что $a$ как функция модулей (значений конденсата) определяется формулой (4)

$$
a_{i}^{\mathrm{D}}=\frac{\partial \mathcal{F}}{\partial a_{i}} .
$$

Тогда, используя свойство дифференциала $d S$, состоящее в том, что его вариации по модулям голоморфны, можно также вычислять матрицу констант связи в калибровочной теории

$$
T_{i j}(u)=\frac{\partial^{2} \mathcal{F}}{\partial a_{i} \partial a_{j}} .
$$

Во-вторых, как было показано в [17], интегралы (4) определяют спектр стабильных состояний в теории, которые насышают предел Богомольного-Прасада-Зоммерфельда (БПС). К примеру, формула для БПС-спектра в теории с группой $S U(2)$ имеет вид

$$
M_{n, m}=\left|n a(u)+m a^{\mathrm{D}}(u)\right|,
$$

где квантовые числа $n, m$ соответствуют “электрическим" и “магнитным" состояниям.

В работе [18] было показано, что каждое решение Виттена-Зайберга может быть ассоциировано с интегрируемой системой с конечным числом степеней свободы, причем можно непосредственно исходя из интегрируемых данных построить препотенщиал.

Действительно, с теорией Виттена-Зайберга ассоциируется следующий набор данных [17]:

а) двумерная риманова поверхность $\mathcal{C}$;

б) пространство модулей $\mathcal{M}$ (кривых $\mathcal{C}$ ), пространство модулей вакуумов калибровочной теории;

в) мероморфиая 1-форма $d S$ на $\mathcal{C}$.

В точности эти исходные данные могут быть естественно описаны в рамках интегрируемой системы, отвечаюшей данной теории Виттена-Зайберга [18]-[20]. 
С этой целью мы введем затравочную спектральную кривую $E$, а именно тор $y^{2}=$ $x^{3}+g_{2} x^{2}+g_{3}$ для ультрафиолетово-конечных калибровочных теорий с ассоциированной голоморфной 1-формой $d \omega=d x / y$. Эта затравочная спектральная кривая для асимптотически свободных теорий вырождается (при размерной трансмутации) в сферу с двумя выколотыми точками (кольцо): $x \rightarrow w+1 / w, y \rightarrow w-1 / w, d \omega=d w / w$. На этой затравочной кривой задан матрично-значный оператор Лакса $L(x, y)$. Соответствующая одетая спектральная кривая $\mathcal{C}$ определяется формулой $\operatorname{det}(L-\lambda)=0$.

Эта спектральная кривая является разветвленным накрытием $E$, заданным уравнением

$$
\mathcal{P}(\lambda ; x, y)=0 .
$$

В случае калибровочной группы $G=S U\left(N_{c}\right)$ функция $\mathcal{P}$ является полиномом степени $N_{c}$ по $\lambda$.

Таким образом, у нас имеется спектральная кривая $\mathcal{C}$, причем пространство модулей $\mathcal{M}$ спектральных кривых дается просто коэффициентами полинома $\mathcal{P}$. Третьим важным ингредиентом конструкции является производящая 1-форма $d S \cong \lambda d \omega$, мероморфнаяна $\mathcal{C}$ ( “” обозначает равенство по модулю полных производных). С точки зрения интегрируемой системы это просто укороченное действие $p d q$ вдоль нестягиваемых контуров на гамильтоновых торах. Это означает, что переменные $a_{i}$ в $(4)$ являются не чем иным, как переменными действия в интегрируемой системе. Определяющим свойством $d S$ является то, что его производные по модулям (точкам ветвления) являются голоморфными дифференциалами на спектральной кривой. Это, в частности, означает, что

$$
\frac{\partial d S}{\partial a_{i}}=d \omega_{i}
$$

где $d \omega_{i}$ - канонические голоморфные дифференциалы ${ }^{2)}$. Интегрируя эту формулу по $B$-циклам и используя равенство $a^{\mathrm{D}}=\partial \mathcal{F} / \partial a$, можно непосредственно получить (6).

До сих пор мы не упоминали материю. Для того чтобы включить гипермультиплеты материи, нужно просто рассмотреть поверхность $\mathcal{C}$ с выколотыми точками. Тогда массы гипермультиплетов будут пропорциональны вычетам $d S$ в этих точках, a пространство модулей должно быть расширено так, чтобы включить эти массовые модули. Все остальные формулы останутся по сути те же.

Препотенциал $\mathcal{F}$ и другие “физические” величины определены в терминах когомологического класса $d S$ формулами (4), (5). Заметим, что формула (6) позволяет идентифицировать препотенциал с логарифмом $\tau$-функции иерархии Уизема [21]: $\mathcal{F}=\ln \tau$. Это иллюстрирует, как другая, уиземовская интегрируемость также возникает в теориях Виттена-Зайберга [22]. На самом деле это явление весьма общее: в матричных моделях

${ }^{2)}$ То есть дифференциалы, удовлетворяющие условиям

$$
\oint_{A_{i}} d \omega_{j}=\delta_{i j}, \quad \oint_{B_{i}} d \omega_{j}=T_{i j}
$$


уиземовская интегрируемость тоже наблюдается [23]. Однако никакой ясной причины этому до сих пор не найдено.

Отметим, что теория Виттена-Зайберга обладает еше более интересными свойствами. Одно из них - это то, что препотенциал $\mathcal{F}$ удовлетворяет некоторому набору крайне нелинейных уравнений, называемых уравнениями ассоциативности (или уравнениями Виттена-Дайкграафа-Верлинде-Верлинде) [24].

\section{5. ДУАЛЬНОСТЬ В ТЕОРИИ ВИТТЕНА-ЗАЙБЕРГА}

Для того чтобы выявить еше одно интересное свойство теории Виттена-Зайберга, нужно взглянуть на полную таблицу $N=2$ суперсимметричных калибровочных теорий в различных измерениях и с различными гипермультиплетами материи [19]. Ограничиваясь теориями с одним присоединенным мультиплетом и меняя количество измерений, мы воспроизводим различные члены дуального интегрируемого семейства Калоджеро-Руджинарса [25]. К примеру, пертурбативный предел четырехмерной теории описывается тригонометрической системой Калоджеро, дуальной некоторому вырожденному пертурбативному пределу пятимерной теории, описываемому рациональной системой Руджинарса. В то же время полный учет непертурбативных поправок требует эллиптических моделей ${ }^{3)}$ и, в конце концов, знания наиболее общего (самодуального) члена семейства - дважды эллиптической системы [26], [27]. Эта система описывает шестимерную (непертурбативную) калибровочную теорию, компактифицированную на двумерный тор, которая была получена только с использованием аргумента (само) дуальности.

Чтобы лучше понять, что эта дуальность значит, мы развиваем далее подход [2], который одновременно позволит понять причину возникновения классической интегрируемости (потоков по константам связи) в квантовых системах, которую мы обсуждали в разделах 2 и 3 . Между тем, мы найдем, что понятие (само)дуальности не слишком ограничивает теорию. Более того, сушествует множество самодуальных систем. Содержательность самодуальности семейства Калоджеро-Руджинарса проявляется скорее в контексте теоретико-групповой интерпретации этого семейства, но не выделяет его среди других интегрируемых систем.

Для прояснения основной идеи подхода [2] мы начнем с простой интегрируемой механической системы ${ }^{4)}$. Интегрируемая система с $N$ координатами $q_{i}$ и $N$ импульсами $p_{i}$

\footnotetext{
3) Заметим, что система, дуальная полной непертурбативной четырехмерной теории, - это $n е p$ турбативная шестимерная теория, компактифицированная на двумерный тор [19], т.е. учет непертурбативных инстантонных поправок эфффективно дуален учету мод Калуцы-Клейна при компактификации на двумерный тор.

4) Мы не касаемся здесь вопроса о том, насколько важна в этом случае интегрируемость на всем фазовом пространстве. Действительно, поскольку наше рассмотрение локально, можно ожидать, что нам понадобится только $N$ гамильтонианов в инволюции, заданных локально, на части фазового пространства. Для простоты мы игнорируем здесь этот вопрос и просто предполагаем, что наша система вполне интегрируема.
} 
характеризуется существованием $N$ гамильтонианов $H_{i}(\vec{p}, \vec{q})$, находящихся в инволюции,

$$
\left\{H_{i}, H_{j}\right\}=\frac{\partial H_{i}}{\partial \vec{p}} \frac{\partial H_{j}}{\partial \vec{q}}-\frac{\partial H_{j}}{\partial \vec{p}} \frac{\partial H_{i}}{\partial \vec{q}}=0 .
$$

Для такой системы можно рассмотреть каноническое преобразование, при котором эти гамильтонианы становятся новыми переменными импульсного или координатного типа. Это преобразование (инфинитезимальной версией которого является определенный гамильтонов поток) будет далее основным предметом обсуждения.

Для конкретности рассмотрим однопараметрическое семейство интегрируемых моделей, параметризованных единственной константой связи $g$ такой, что модель является невзаимодействующей при $g=0$. Это значит, что при $g=0$ гамильтонианы $H_{i}^{(0)}(\vec{p})=$ $\left.H_{i}(\vec{p}, \vec{q} \mid g)\right|_{g=0}$ являются функциями только импульсов $\vec{p}$, хотя при обычных выборах гамильтонианов в конкретных приложениях эти функции могут быть нетривиальными. Типичные примеры таких гамильтонианов

$$
H_{k}^{(0)}(\vec{p})=\sum_{i=0}^{N} p_{i}^{k}, \quad H_{k}^{(0)}(\vec{p})=\sum_{I:|I|=k} \prod_{i \in I} e^{p_{i}}
$$

для $p$-рациональных и $p$-тригонометрических моделей, соответственно, а также некоторые эллиптические функции $\vec{p}$ для их тригонометрических обобщений.

Адекватное определение новых канонических переменных $\vec{P}_{g}=\vec{P}(\vec{p}, \vec{q} \mid g)$ и $\vec{Q}_{g}=$ $\vec{Q}(\vec{p}, \vec{q} \mid g)$ можно записать $\left.{ }^{5}\right)$ в виде [26]

$$
H_{i}^{(0)}(\vec{P})=H_{i}(\vec{p}, \vec{q} \mid g), \quad \widetilde{H}_{i}^{(0)}(\vec{q})=\widetilde{H}_{i}(\vec{Q}, \vec{P} \mid g),
$$

где $\widetilde{H}_{i}(\vec{p}, \vec{q} \mid g)$ определяет гамильтонианы дуальной интегрируемой системы. Это точное определение того, что такое дуальность в механической интегрируемой системе, в частности в теории Виттена-Зайберга. Его не следует путать с “физической” электромагнитной дуальностью в теории Виттена-Зайберга [17], которая является дуальностью между “электрическими” и “магнитными” состояниями в (7).

Заметим, что в (9) гамильтонианы зависят от "одетых" переменных $\vec{P}$ и $\vec{Q}$ и, более того, $\vec{P}$ и $\vec{Q}$ меняются местами. Форма дуальных гамильтонианов диктуется требованием, чтобы новые переменные $\vec{P}$ и $\vec{Q}$ были каноническими, т.е.

$$
\sum_{i} d P_{i} \wedge d Q_{i}=\sum_{i} d p_{i} \wedge d q_{i}
$$

а скобки Пуассона имели вид

$$
\{\cdot, \cdot\}=\sum_{i}\left(\frac{\partial}{\partial p_{i}} \otimes \frac{\partial}{\partial q_{i}}-\frac{\partial}{\partial q_{i}} \otimes \frac{\partial}{\partial p_{i}}\right)=\sum_{i}\left(\frac{\partial}{\partial P_{i}} \otimes \frac{\partial}{\partial Q_{i}}-\frac{\partial}{\partial Q_{i}} \otimes \frac{\partial}{\partial P_{i}}\right) .
$$

\footnotetext{
5) Далее мы часто опускаем индекс $g$, подразумевая, что прописные буквы $P$ и $Q$ всегда обозначают "одетые" импульсы и координаты $P_{g}$ и $Q_{g}$.
} 
В разделе 9 мы увидим, что это определение дуальных гамильтонианов действительно приводит к дуальности тригонометрической системы Калоджеро рациональной системе Руджинарса, самодуальности тригонометрической системы Руджинарса и т.д. Однако это определение подразумевает, что любая интегрируемая система имеет дуальную, т.е. дуальность различных членов семейства Калоджеро-Руджинарса не означает ничего специального об этих интегрируемых системах. И только лишш их интерпретация в теоретико-групповых терминах придает этой дуальности особый интерес. Однако здесь мы не останавливаемся на этом вопросе, а обсуждаем лишь общие свойства интегрируемых систем.

\section{6. ИНТЕГРИРУЕМОСТЬ И ПОТОКИ ПО КОНСТАНТАМ СВЯЗИ: ОБШАЯ ТЕОРИЯ}

Соотношения (9) определяют $\vec{P}_{g}$ как функции $\vec{p}, \vec{q}$ и константы связи $g$. Особую важность и интерес представляет инфинитезимальная версия этого канонического преобразования, рассматриваемого как гамильтонов поток вдоль направления $g$ в пространстве констант связи. Такое преобразование генерируется некоторым новым гамильтонианом $T(\vec{p}, \vec{q} \mid g)$ согласно правилу

$$
\begin{gathered}
\frac{\partial \vec{P}_{g}}{\partial g}=\left\{T\left(\vec{P}_{g}, \vec{Q}_{g} \mid g\right), \vec{P}_{g}\right\}=-\frac{\partial T}{\partial \vec{Q}_{g}} \\
\frac{\partial \vec{Q}_{g}}{\partial g}=\left\{T\left(\vec{P}_{g}, \vec{Q}_{g} \mid g\right), \vec{Q}_{g}\right\}=\frac{\partial T}{\partial \vec{P}_{g}} .
\end{gathered}
$$

Этот новый гамильтониан не находится в инволюции со старыми, но преврашает их в некоторьй новый набор гамильтонианов в инволюции.

Более того, вместо рассмотрения некоторого заранее заданного семейства интегрируемых систем можно использовать любую функцию $T(\vec{p}, \vec{q} \mid g)$, для того чтобы определить целое однопараметрическое семейство интегрируемых систем, хотя явная конструкция соответствующих гамильтонианов в инволюции редко возможна. Далее, многопараметрическое семейство интегрируемых систем может быть сгенерировано подоб-

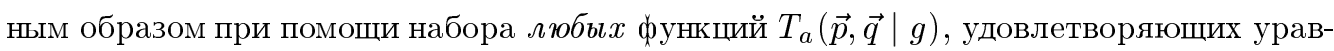
нениям самосогласованности (уравнениям Уизема)

$$
\frac{\partial T_{b}}{\partial g_{a}}-\frac{\partial T_{a}}{\partial g_{b}}+\left\{T_{a}, T_{b}\right\}=0 .
$$

Заметим, что после квантования системы образуется семейство квантовых операторов $\widehat{T}_{a}\left(\hat{\vec{p}}, \hat{\vec{q}} \mid\left\{g_{b}\right\}\right)$, удовлетворяюших условию нулевой кривизны в пространстве констант связи

$$
\left[\frac{\partial}{\partial g_{a}}-\widehat{T}_{a}(\hat{\vec{p}}, \hat{\vec{q}}), \frac{\partial}{\partial g_{b}}-\widehat{T}_{b}(\hat{\vec{p}}, \hat{\vec{q}})\right]=0 .
$$

Это может служить некоторым объяснением возникновения классической интегрируемости (уравнения нулевой кривизны) в квантовых интегрируемых системах, которые 
мы обсуждали в предыдущих разделах. В частности, было бы интересно в рамках формализма данной статьи изучить случаи, описанные в разделах 2 и 3 и ассоциированные с интегрируемыми механическими системами с бесконечным числом степеней свободы.

Вариация констант связи в дуальных интегрируемых системах задается дуальным гамильтонианом $\widetilde{T}(\vec{p}, \vec{q} \mid g)$ : для

$$
\widetilde{H}_{i}^{(0)}(\widetilde{\vec{P}})=\widetilde{H}_{i}(\vec{p}, \vec{q} \mid g)
$$

(и $\left.H_{i}^{(0)}(\vec{q})=H_{i}(\widetilde{\vec{Q}}, \widetilde{\vec{P}} \mid g)\right)$ мы имеем

$$
\begin{aligned}
& \frac{\partial \widetilde{\vec{P}}_{g}}{\partial g}=\left\{\widetilde{T}\left(\widetilde{\vec{P}}_{g}, \widetilde{\vec{Q}}_{g} \mid g\right), \widetilde{\vec{P}}_{g}\right\}=-\frac{\partial \widetilde{T}}{\partial \widetilde{\vec{Q}}_{g}} \\
& \frac{\partial \widetilde{\vec{Q}}_{g}}{\partial g}=\left\{\widetilde{T}\left(\widetilde{\vec{P}}_{g}, \widetilde{\vec{Q}}_{g} \mid g\right), \widetilde{\vec{Q}}_{g}\right\}=\frac{\partial \widetilde{T}}{\partial \widetilde{\vec{P}}_{g}} .
\end{aligned}
$$

Соотношение между $\widetilde{T}(\vec{p}, \vec{q} \mid g)$ и $T(\vec{p}, \vec{q} \mid g)$ становится особенно простым в любой самодуальной точке (где $\left.\widetilde{H}_{i}\left(\vec{p}, \vec{q} \mid g_{\mathrm{SD}}\right)=H_{i}\left(\vec{p}, \vec{q} \mid g_{\mathrm{SD}}\right)\right)$ :

$$
\widetilde{T}\left(\vec{p}, \vec{q} \mid g_{\mathrm{SD}}\right)=T\left(\vec{q}, \vec{p} \mid g_{\mathrm{SD}}\right)
$$

Как прямое следствие, любая симметричная функция $T(\vec{p}, \vec{q} \mid g)=T(\vec{q}, \vec{p} \mid g)$ определяет однопараметрическое семейство самодуальных интегрируемых систем. Следовательно, не просто дуальность, но даже и свойство самодуальности моделей семейства Калоджеро-Руджинарса (рациональной системы Калоджеро, тригонометрической системы Руджинарса и дважды эллиптической системы) никоим образом не является специфическим. Мы снова приходим к утверждению, что (само)дуальность этого семейства интересна только в теоретико-групповом контексте.

Заметим, что задача восстановления гамильтониана начиная с произвольно заданного $T(\vec{p}, \vec{q} \mid g)$ решается не так уж просто. Скажем, можно построить ряд теории возмущений по $g$. В самодуальной точке для системы с одной степенью свободы разложение имеет вид

$$
T(p, q \mid g) \equiv \sum_{i=1} T_{i}(p, q) g^{i-1}, \quad P=H(p, q)=p+\sum_{i=1} \Phi_{i}(p, q) g^{i}
$$

и т.д., так что получается

$$
\Phi_{1}(p, q)=\partial_{q} T_{1}(p, q), \quad \Phi_{2}(p, q)=\partial_{q} T_{2}(p, q)+\cdots
$$


Окончательно симметричная часть от $\partial_{p} \Phi_{i}(p, q)=\partial_{q, p}^{2} T_{i}(p, q)+\cdots$ задается независимо в любом порядке, в то время как антисимметричная часть фиксируется $\Phi_{i}$ в низших порядках:

$$
\begin{gathered}
\Phi_{1}^{\prime}(p, q)-\Phi_{1}^{\prime}(q, p)=0, \\
\Phi_{2}^{\prime}(p, q)-\Phi_{2}^{\prime}(q, p)=\Phi_{1}^{\prime \prime}(p, q) \Phi_{1}(p, q)-(p \leftrightarrow q), \\
\Phi_{3}^{\prime}(p, q)-\Phi_{3}^{\prime}(q, p)=\Phi_{1}^{\prime \prime}(p, q) \Phi_{2}(p, q)+\Phi_{2}^{\prime \prime}(p, q) \Phi_{1}(p, q)-\frac{1}{2} \Phi_{1}^{\prime \prime \prime}(p, q) \Phi_{1}^{2}(p, q)- \\
-\Phi_{1}^{\prime \prime}(p, q) \Phi_{1}^{\prime}(p, q) \Phi_{1}(p, q)-(p \leftrightarrow q), \quad \ldots,
\end{gathered}
$$

где все производные берутся по отношению к первой переменной. Тривиальное решение уравнений $(17)$ - это $\Phi_{i}(p, q)=p^{n_{i}+1} q^{n_{i}}$ с произвольньми $\left\{n_{i}\right\}$. Отсюда следует, что гамильтониан $H(p, q)=p f(p q)$ самодуален для любой функции $f$. Действительно, легко проверить, что это так (заметим, что для такого гамильтониана $p q=P Q$ ). В частности, гамильтониан рациональной системы Калоджеро относится к данному классу гамильтонианов.

\section{7. ПРОИЗВОДЯЩИЕ ФУНКЦИИ И КВАНТОВАНИЕ}

Вместе с гамильтонианами $T(\vec{P}, \vec{Q} \mid g)$ можно также рассматривать производяшие функции обсуждаемых канонических преобразований, подобные $S(\vec{Q}, \vec{q} \mid g)$ или полученной из нее преобразованием Лежандра $F(\vec{P}, \vec{q} \mid g)=\vec{P} \vec{Q}-S(\vec{Q}, \vec{q} \mid g)$, так, что

$$
-\vec{P}=\frac{\partial S}{\partial \vec{Q}}, \quad \vec{p}=\frac{\partial S}{\partial \vec{q}}
$$

и

$$
T=\frac{\partial S}{\partial g} .
$$

Конечно, для канонических преобразований

$$
\frac{\partial P_{i}}{\partial q_{j}}+\frac{\partial p_{j}}{\partial Q_{i}}=0
$$

как подразумевается в (18). Однако, получая подобное заключение из (19), нужно быть аккуратным: вторые производные по $g$ удовлетворяют уравнению (13), поскольку производные по $g$ берутся при постоянных $\vec{P}_{g}$ и $\vec{Q}_{g}$, которые сами зависят от $g$.

Аналогично

$$
-\vec{Q}=\frac{\partial F}{\partial \vec{P}}, \quad \vec{p}=\frac{\partial F}{\partial \vec{q}} .
$$

В самодуальных точках $F\left(\vec{P}, \vec{q} \mid g_{\mathrm{SD}}\right)=F\left(\vec{q}, \vec{P} \mid g_{\mathrm{SD}}\right)$ является симметричной функцией.

Заметим, что

$$
\frac{\partial F}{\partial g}=T
$$


(с постоянными $p$ и $Q$ ), т.е. $T$ в некотором смысле является более инвариантной величиной, чем $S$ и $F$, не зависящей от выбора независимых переменных. Другой способ увидеть это - рассмотреть поток из интегрируемой системы с константой связи $g_{1}$ в ту же самую систему с константой связи $g_{2}$. В этом случае гамильтониан $T$ зависит только от $g_{2}$, но не от $g_{1}$, в то время как производящие функции зависят и от $g_{1}$, и от $g_{2}$.

В сущности, будучи выражен в терминах $\vec{Q}$ и $\vec{q}$ (вместо $\vec{P}$ и $\vec{Q}$ ), гамильтониан принимает вид $T=\partial S / \partial g$. Следовательно, $e^{i S}$ можно рассматривать как нечто вроде оператора эволюции (ядра) в пространстве констант связи, который обеспечивает каноническое преобразование из свободной системы в интегрируемую. После квантования это может быть символически представлено как

$$
e^{i \widehat{S}}(\vec{Q}, \vec{q} \mid g)=\bigoplus_{\lambda}\left|\psi_{\lambda}^{(0)}(\vec{Q})\right\rangle c_{\vec{\lambda}}\left\langle\psi_{\lambda}(\vec{q})\right|,
$$

где $\left|\psi_{\lambda}\right\rangle$ и $\left|\psi_{\lambda}^{(0)}\right\rangle$ - собственные функции системы и $c_{\vec{\lambda}}-$ некоторые коэффициенты, зависящие от спектрального параметра $\vec{\lambda}$. Оператор эволюции удовлетворяет квантовой версии уравнения (9)

$$
H^{(0)}\left(\frac{\partial}{\partial \vec{Q}}\right)=e^{-i \widehat{S}(\vec{Q}, \vec{q} \mid g)} H\left(\frac{\partial}{\partial \vec{q}}, \vec{q} \mid g\right) e^{i \widehat{S}(\vec{Q}, \vec{q} \mid g)} .
$$

Поскольку собственные функции $\left|\psi_{\lambda}^{(0)}(\vec{Q})\right\rangle$ свободной системы являются экспонентами от спектрального параметра $\vec{\lambda}$, одетые собственные функции $\left|\psi_{\lambda}(\vec{q})\right\rangle$-это фурье-образы оператора эволюции $e^{i \widehat{S}(\vec{Q}, \vec{q})}$ :

$$
\psi_{\lambda}(\vec{q}) \sim \int e^{i \widehat{S}(\vec{Q}, \vec{q})} e^{i \lambda \vec{Q}} d \vec{Q}
$$

Аналогично получаем

$$
\psi_{\lambda}(\vec{q}) \sim \int e^{i \widehat{F}(\vec{P}, \vec{q})} \delta(\lambda-\vec{P}) d \vec{P} \sim e^{i \widehat{F}(\lambda, \vec{q})} .
$$

Заметим, что в решениях уравнения (10) имеется свобода сдвига $Q_{i}$ на произвольную функцию переменной $P_{i}$. Этот сдвиг имеет весьма сложный вид для производящей функции $S$, но для функции $F$ он сводится к добавлению члена, зависяшего только от $P_{i}$. В квантовом случае эта неоднозначность в определении $F$ просто сводится к нормировке собственной функции.

Можно также рассмотреть квантовый аналог $T(p, q \mid g)$, который является гамильтонианом, задаюшим уравнение Шредингера по константе связи

$$
\frac{\partial \psi}{\partial g}=i \widehat{T} \psi
$$

Следовательно, волновая функция $\psi$ может быть также реализована при помощи интеграла по путям по переменным фазового пространства $P(g), Q(g)$.

Производящие функции $S(Q, q), F(P, q)$ и $T(p, q)$ удовлетворяют квазиклассической версии приведенных выше соотношений (когда можно пренебречь многократными производными от $S, F$ и $T$ ). 


\section{8. КОНКРЕТНЫЕ ПРИМЕРЫ}

Для того чтобы проиллюстрировать рассмотрение предыдущих разделов, мы кратко обсудим здесь несколько явных примеров (подробнее см. [2]), ограничившись только системами с одной степенью свободы. Начнем с простейшего примера гармонического осциллятора. В этом случае

$$
H(p, q \mid \omega)=\frac{1}{2}\left(p^{2}+\omega^{2} q^{2}\right)
$$

Пусть частота $\omega$ играет роль константы связи, так что $H^{(0)}(p)=p^{2} / 2$. Тогда

$$
P=\sqrt{p^{2}+\omega^{2} q^{2}}, \quad Q=\frac{\sqrt{p^{2}+\omega^{2} q^{2}}}{\omega} \operatorname{arctg} \frac{\omega q}{p} .
$$

Обратное преобразование имеет вид

$$
p=P \cos \frac{\omega Q}{P}, \quad q=\frac{P}{\omega} \sin \frac{\omega Q}{P},
$$

т.е. гамильтониан дуального потока

$$
\widetilde{H}(\tilde{p}, \tilde{q} \mid \omega)=\frac{\tilde{q}}{\omega} \sin \frac{\omega \tilde{p}}{\tilde{q}} .
$$

(Подчеркнем, что при этом определении $\widetilde{H}^{(0)}(p)=p$, а также заметим, что дуальность нарушает счет размерностей, если приписывать $p$ и $q$ их обычные размерности, так что не должно быть сюрпризом, что $\omega q / p$ в $H$ заменяется на $\omega \tilde{p} / \tilde{q}$ в $\widetilde{H}$. Конечно, $\tilde{p}$ и $\tilde{q}$ суть не что иное, как $Q_{\omega}$ и $P_{\omega}$.)

Генератор $\omega$-эволюции

$$
T=\frac{P^{2}}{4 \omega^{2}} \sin \frac{2 \omega Q}{P}-\frac{P Q}{2 \omega}=\frac{p q-P Q}{2 \omega} .
$$

Для дуальной системы аналогично имеем

$$
\widetilde{T}=\frac{-\omega \widetilde{P} \widetilde{Q}+\left(\widetilde{Q}^{2}+\omega^{2} \widetilde{P}^{2}\right) \operatorname{arctg}(\omega \widetilde{P} / \widetilde{Q})}{2 \omega^{2}}=\frac{\tilde{p} \tilde{q}-\widetilde{P} \widetilde{Q}}{2 \omega}=\frac{P Q-p q}{2 \omega}=-T .
$$

Производящая функция

$$
\begin{aligned}
F(P, q \mid \omega) & =\int p d q=\int \sqrt{P^{2}-\omega^{2} q^{2}} d q= \\
& =\frac{1}{2} q \sqrt{P^{2}-\omega^{2} q^{2}}+\frac{P^{2}}{2 i \omega} \ln \left(i \frac{\omega q}{P}+\sqrt{1-\frac{\omega^{2} q^{2}}{P^{2}}}\right),
\end{aligned}
$$

так что соотношение (26) в квазиклассическом приближении принимает вид

$$
e^{i F(\lambda, q)}=\left(i \frac{\omega q}{\lambda}-\sqrt{1-\frac{\omega^{2} q^{2}}{\lambda^{2}}}\right)^{\lambda^{2} /(2 \omega)} e^{(i q / 2) \sqrt{\lambda^{2}-\omega^{2} q^{2}}} \sim e^{-\omega q^{2} / 2} H e_{\nu}(\sqrt{\omega} q),
$$


где $\lambda^{2} /(2 \omega)=\nu+1 / 2$ и полиномы Эрмита, удовлетворяюшие равенству

$$
\left(-\partial_{x}^{2}+x^{2}\right) e^{-x^{2} / 2} H e_{\nu}(x)=(2 \nu+1) e^{-x^{2} / 2} H e_{\nu}(x),
$$

даются обратным преобразованием Лапласа производящей функции $e^{-x^{2} / 4+t / x}$ :

$$
H e_{\nu}(x) \sim \int \frac{d t}{t^{\nu+1}} e^{-t^{2} / 4+t x}
$$

Взяв этот интеграл методом перевала, получим

$$
H e_{\nu}(x) \sim\left(x-\sqrt{x^{2}-2(\nu+1)}\right)^{\nu+1} e^{x^{2} / 2+(x / 2) \sqrt{x^{2}-2(\nu+1)}},
$$

что согласуется при больших $\nu$ с соотношением (34).

Для одной частишы (одной степени свободы) любая динамика является интегрируемой и любой гамильтониан канонически эквивалентен свободному. Следовательно, имеет смысл изучить общий гамильтониан. Для $H=\sqrt{p^{2}+g^{2} V(q)}$ дуальный гамильтониан - это $q=\widetilde{H}(P, Q)$. Условие каноничности тогда дает

$$
\frac{\partial \widetilde{H}(P, Q)}{\partial Q}=\frac{p(P, Q)}{P} .
$$

Следовательно, дуальный гамильтониан может быть получен решением уравнения

$$
Q=\int^{q} \frac{d \xi}{\sqrt{1-g^{2} V(\xi) / P^{2}}}
$$

по отношению к $q$. Взяв теперь производную от $P=\sqrt{p^{2}+g^{2} V(q)}$ по $g$, получим

$$
-\frac{\partial T}{\partial Q}=\frac{g}{P} V(q(P, Q))
$$

где $q(P, Q)$ дается (39).

\section{9. СЕМЕЙСТВО КАЛОДЖЕРО-РУДЖИНАРСА}

В заключение приведем также результаты для рациональных и тригонометрических членов семейства Калоджеро-Руджинарса (которые описывают различные пределы теории Виттена-Зайберга с присоединенным гипермультиплетом материи и в различных размерностях).

1.Рационально-рациональный случай (рациональная модель Калоджеро). Единственный гамильтониан в этом случае $-H=\left(p^{2}+g^{2} / q^{2}\right) / 2$, и, таким образом, $H^{0}(p)=$ $p^{2} / 2, \mathrm{a}$

$$
P^{2}=p^{2}-\frac{g^{2}}{q^{2}}, \quad q^{2}=Q^{2}-\frac{g^{2}}{P^{2}}
$$


Рациональная модель Калоджеро самодуальна. Поток, генерированный гамильтонианом:

$$
T(p, q ; g)=\frac{1}{2} \ln \frac{p q-g}{p q+g}=\frac{1}{2} \ln \frac{P Q-g}{P Q+g} .
$$

Это, очевидно, симметричная функция $p$ и $q$, т.е. $T_{\mathrm{D}}(p, q)=T(p, q)$, как и должно быть для самодуальной системы. Производящая функция

$$
\begin{gathered}
S=g \operatorname{arch} \frac{Q}{q}=g \ln \frac{Q-\sqrt{Q^{2}-q^{2}}}{q}=\frac{g}{2} \ln \frac{Q-\sqrt{Q^{2}-q^{2}}}{Q+\sqrt{Q^{2}-q^{2}}} \\
\frac{\partial S(Q, q \mid g)}{\partial g}=T(p(Q, q), q \mid g) .
\end{gathered}
$$

В этом случае $S$ - простая линейная функция $g$ и $\partial S / \partial g=S / g$. Подобным образом

$$
\begin{gathered}
F(P, q)=\sqrt{P^{2} q^{2}+g^{2}}+\frac{g}{2} \frac{\ln \sqrt{P^{2} q^{2}+g^{2}}-g}{\sqrt{P^{2} q^{2}+g^{2}}+g}, \\
\frac{\partial F(P, q \mid g)}{\partial g}=T(p(P, q), q \mid g) .
\end{gathered}
$$

В соответствии с (25)

$$
\psi_{\lambda}(q) \sim \int e^{i S(q, Q \mid g)} e^{i Q \lambda} d Q \sim q J_{i g}(i \lambda q),
$$

где $J_{g}(x)$ - функция Бесселя. Точная квантовая волновая функция, удовлетворяющая уравнению

$$
\left(-\partial_{x}^{2}+\frac{g^{2}}{x^{2}}\right) \psi_{\lambda}(x)=\lambda^{2} \psi_{\lambda}(x),
$$

- это функция $\sqrt{x} J_{\nu}(i \lambda x), \nu^{2}=-g^{2}+1 / 4$. Она совпадает с (45) в квазиклассическом приближении.

2. Рационально-тригонометрический случай (тригонометрическая модель Калоджеро). В этом случае

$$
\begin{gathered}
P^{2}=2 H=p^{2}-\frac{g^{2}}{\operatorname{sh}^{2} q} \\
\operatorname{ch}^{2} q=\widetilde{H}^{2}=\operatorname{ch}^{2} Q\left(1-\frac{g^{2}}{P^{2}}\right), \\
T=\frac{1}{2} \ln \frac{P \operatorname{th} Q-g}{P \operatorname{th} Q+g}=\frac{1}{2} \ln \frac{p \operatorname{th} q-g}{p \operatorname{th} q+g},
\end{gathered}
$$

$S$ все еще является простой линейной функцией $g$ :

$$
S=g \operatorname{arsh}\left(\frac{\operatorname{sh} Q}{\operatorname{sh} q}\right)
$$


3. Тригонометрически-рациональный случай (рациональная модель Руджинарса). В этом случае

$$
\begin{gathered}
\operatorname{ch}^{2} P=H^{2}=\operatorname{ch}^{2} p\left(1-\frac{\operatorname{sh}^{2} \epsilon}{q^{2}}\right), \\
q^{2}=2 \widetilde{H}=Q^{2}-\frac{\operatorname{sh}^{2} \epsilon}{\operatorname{sh}^{2} P}, \\
T=\frac{1}{2} \ln \frac{Q \operatorname{th} P-\operatorname{th} \epsilon}{Q \operatorname{th} P+\operatorname{sh} \epsilon}=\frac{1}{2} \ln \frac{q \operatorname{th} p-\operatorname{th} \epsilon}{q \operatorname{th} p+\operatorname{sh} \epsilon},
\end{gathered}
$$

$S$ уже больше не является простой линейной функцией константы связи $\epsilon$.

4. Тригонометрически-тригонометрический случай (тригонометрическая модель Руджинарса):

$$
\begin{gathered}
\operatorname{ch}^{2} P=H^{2}=\operatorname{ch}^{2} p\left(1-\frac{\operatorname{sh}^{2} \epsilon}{\operatorname{sh}^{2} q}\right), \\
\operatorname{ch}^{2} q=\widetilde{H}^{2}=\operatorname{ch}^{2} Q\left(1-\frac{\operatorname{sh}^{2} \epsilon}{\operatorname{sh}^{2} P}\right), \\
T=\frac{1}{2} \ln \frac{\operatorname{th} P \operatorname{th} Q-\operatorname{th} \epsilon}{\operatorname{th} P \operatorname{th} Q+\operatorname{th} \epsilon}=\frac{1}{2} \ln \frac{\operatorname{th} p \operatorname{th} q-\operatorname{th} \epsilon}{\operatorname{th} p \operatorname{th} q+\operatorname{th} \epsilon} .
\end{gathered}
$$

\section{0. ЗАКЛЮЧЕНИЕ}

Таким образом, начав с обшего описания того, что такое интегрируемость в теориях поля/струн и что могло бы быть причиной ее возникновения, мы затем рассмотрели ряд простейших примеров систем с одной степенью свободы, которые иллюстрируют, как константы связи могут играть роль переменных времени, отвечая гамильтоновым потокам. Однако обобщение этой схемы технически весьма сложно, и до сих пор мы не знаем явных формул для систем со многими степенями свободы. С другой стороны, ясно, что должна существовать схема, которая позволила бы конструировать гамильтоновы потоки по константам связи с использованием только содержащейся в интегрируемой системе информации, подобной оператору Лакса. Такого рода схему можно было бы попытаться угадать, имея какой-нибудь явный пример многочастичной системы. Построение такого примера остается очень важной проблемой.

Следуюшим важным шагом могло бы стать дальнейшее изучение квантового случая. Действительно, мы здесь только вкратце обсудили его, но по сравнению с классическим случаем имеется ряд проблем, которые должны быть разрешены. Прежде всего, это проблема нормального упорядочивания квантовых операторов эволюции и характер зависимости результата от него. Сушествуют также проблемы эффектов дискретных спектров и идентификации спектров при различных значениях констант связи, т.е. точного определения спектрального параметра $\lambda$ таким образом, чтобы уравнение (23) было диагонально по $\lambda$.

Другой интересный вопрос связан с возникновением интегрируемости в квантовых теориях поля. А именно, было бы очень поучительно, следуя обсуждаемой здесь схеме, проследить, как интегрируемость по константам связи теории поля возникает в известных примерах. В частности, можно изучить квантовое нелинейное уравнение Шре-

4 Теоретическая и математическая физика, т. 135, № 3, 2003 г. 
дингера для того, чтобы увидеть, как потоки по различным константам связи приводят к уравнению нулевой кривизны для классического нелинейного уравнения Шредингера [10]. Поскольку окончательньй ответ известен, можно надеяться, что такое вычисление удастся проделать.

Благодарности. Я благодарен А. Морозову за ценные обсуждения и С. Харчеву за чтение настоящей статьи. Работа частично поддержана грантами INTAS (№ 00-561), РФФИ (№ 01-02-17682а), грантом поддержки научных школ (№ 96-15-96798) и грантом Volkswagen-Stiftung.

\section{Список литературы}

[1] А. Морозов. УФН. 1992. Т. 162. C. 83; hep-th/9303139; 1994. Т. 164. C. 3; hep-th/9502091; A. Mironov. Int. J. Mod. Phys. A. 1994. V. 9. P. 4355; hep-th/9312212.

[2] A. Mironov, A. Morozov. Phys. Lett. B. 2002. V. 524. P. 217.

[3] E. Brezin, V. Kazakov. Phys. Lett. B. 1990. V. 236. P. 144; M. Douglas, S. Shenker. Nucl. Phys. B. 1990. V. 335. P. 635; D. Gross, A. Migdal. Phys. Rev. Lett. 1990. V. 64. P. 12.

[4] A. Gerasimov, A. Marshakov, A. Mironov, A. Morozov, A. Orlov. Nucl. Phys. B. 1991. V. 357. P. 565.

[5] M. Тода. Теория нелинейных решеток. М.: Мир, 1984.

[6] A. Mironov, A. Morozov. Phys. Lett. B. 1990. V. 252. P. 47; J. Ambjørn, J. Jurkiewicz, Yu. M. Makeenko. Phys. Lett. B. 1990. V. 251. P. 517; H. Itoyama, Y. Matsuo. Phys. Lett. B. 1991. V. 255. P. 202; F. David. Mod. Phys. Lett. A. 1990. V. 5. P. 1019.

[7] A. Mironov, A. Morozov. Phys. Lett. B. 2000. V. 490. P. 173; hep-th/0005280.

[8] M. Fukuma, H. Kawai, R. Nakayama. Int. J. Mod. Phys. A. 1991. V. 6. P. 1385; Commun. Math. Phys. 1992. V. 143. P. 371.

[9] E. Barouch, B. M. McCoy, T. T. Wu. Phys. Rev. Lett. 1973. V. 31. P. 1409; C. A. Tracy, B. M. McCoy. Phys. Rev. Lett. 1973. V. 31. P. 1500; T. T. Wu, B. M. McCoy, C. A. Tracy, E. Barouch. Phys. Rev. B. 1976. V. 13. P. 316; M. Jimbo, T. Miwa, M. Sato. Publ. RIMS Kyoto Univ. 1978. V. 14. P. 223; 1979. V. 15. P. 201; P. 577; P. 871; P. 1531; D. Bernard, A. LeClair. Nucl. Phys. B. 1994. V. 426. № 3. P. 534; (erratum). 1997. V. 498. P. 619; hep-th/9402144.

[10] A.R. Its, A. G. Izergin, V.E. Korepin, N. A. Slavnov. Int. J. Mod. Phys. B. 1990. V. 4. P. 1003.

[11] A. G. Izergin, D. A. Coker, V. E. Korepin. J. Phys. A. 1992. V. 25. P. 4315.

[12] S. Kharchev, A. Marshakov, A. Mironov, A. Morozov, A. Zabrodin. Phys. Lett. B. 1992. V. 275. P. 311; hep-th/9111037; Nucl. Phys. B. 1992. V. 380. P. 181; hep-th/9201013.

[13] S. Kharchev, A. Marshakov, A. Mironov, A. Morozov. Int. J. Mod. Phys. A. 1995. V. 10. P. 2015; hep-th/9312210; A. Mironov, A. Morozov, G. W. Semenoff. Int. J. Mod. Phys. A. 1996. V. 11. P. 5031; hep-th/9404005.

[14] В. Книжник. УФН. 1989. Т. 159. С. 401.

[15] G. Akemann, P. H. Damgaard. Nucl. Phys. B. 2000. V. 576. P. 597; hep-th/9910190; H. W. Braden, A. Mironov, A. Morozov. Phys. Lett. B. 2001. V. 514. P. 293.

[16] A. Morozov. Identities between quantum field theories in different dimensions. hep-th/9810031.

[17] N. Seiberg, E. Witten. Nucl. Phys. B. 1994. V. 426. P. 19; (erratum). 1994. V. 430. P. 485; hep-th/9407087; Nucl. Phys. B. 1994. V. 431. P. 484; hep-th/9408099.

[18] A. Gorsky, I. Krichever, A. Marshakov, A. Mironov, A. Morozov. Phys. Lett. B. 1995. V. 355. P. 466; hep-th/9505035. 
[19] H.W. Braden, I. M. Krichever (Eds.). Integrability: The Seiberg-Witten and Whitham Equations. Amsterdam: Gordon and Beach, 2000; A. Gorsky, A. Mironov. Integrable many-body systems and gauge theories. hep-th/0011197.

[20] H. Itoyama, A. Morozov. Nucl. Phys. B. 1996. V. 477. P. 855; hep-th/9511126; Nucl. Phys. B. 1997. V. 491. P. 529; hep-th/9512161; New matrix model solutions to the Kac-Schwarz problem. hep-th/9601168.

[21] I. Krichever. Commun. Pure Appl. Math. 1994. V. 47. P. 437; B. Dubrovin. Nucl. Phys. B. 1992. V. 379. P. 627; Geometry of 2d topological field theories. hep-th/9407018.

[22] A. Gorsky, A. Marshakov, A. Mironov, A. Morozov. Nucl. Phys. B. 1998. V. 527. P. 690; hep-th/9802007.

[23] S. Kharchev, A. Marshakov, A. Mironov, A. Morozov. Mod. Phys. Lett. A. 1993. V. 8. Р. 1047; С. Харчев, А. Мариаков, А. Миронов, А. Морозов. ТМФ. 1993. Т. 95. № 2. C. 280 ; hep-th/9208046.

[24] A. Marshakov, A. Mironov, A. Morozov. Phys. Lett. B. 1996. V. 389. P. 43; hep-th/9607109; Mod. Phys. Lett. A. 1997. V. 12. P. 773; hep-th/9701014; Int. J. Mod. Phys. A. 2000. V. 15. P. 1157; hep-th/9701123.

[25] S. N. Ruijsenaars. Commun. Math. Phys. 1988. V. 115. P. 127; O. Babelon, D. Bernard. Phys. Lett. B. 1993. V. 317. P. 363; V. Fock. Three remarks on group invariants related to flat connections. In: Geometry and Integrable Models. Eds. P. Pyatov, S. Solodukhin. Singapore: World Scientific, 1995. P. 20; V. Fock, A. Rosly. Amer. Math. Soc. Transl. 1999. V. 191. P. 67; math.QA/9802054; V. Fock, A. Gorsky, N. Nekrasov, V. Roubtsov. JHEP. 2000. V. 0007. P. 028; A. Gorsky, V. Rubtsov. Dualities in integrable systems: geometric aspects. hep-th/0103004.

[26] H. Braden, A. Marshakov, A. Mironov, A. Morozov. Nucl. Phys. B. 2000. V. 573. P. 553; hep-th/9906240; А. Д. Миронов. ТМФ. 2001. Т. 129. С. 327; hep-th/0104253.

[27] A. Mironov, A. Morozov. Phys. Lett. B. 2000. V. 475. P. 71; hep-th/9912088; Double elliptic systems: problems and perspectives. hep-th/0001168; H. Braden, A. Gorsky, A. Odesskii, V. Rubtsov. Nucl. Phys. B. 2002. V. 633. P. 414; hep-th/0111066. 